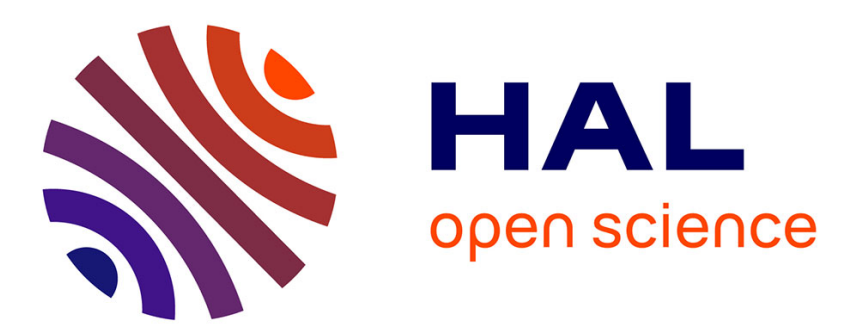

\title{
Étude de quelques facteurs de variation de la fécondité chez Trichogramma maidis Pintureau et Voegele (Hym. Trichogrammatidae)
}

\author{
Bernard Pintureau, Manuel Babault, Jean Voegele
}

\section{To cite this version:}

Bernard Pintureau, Manuel Babault, Jean Voegele. Étude de quelques facteurs de variation de la fécondité chez Trichogramma maidis Pintureau et Voegele (Hym. Trichogrammatidae). Agronomie, 1981, 1 (4), pp.315-322. hal-00884260

\section{HAL Id: hal-00884260 \\ https://hal.science/hal-00884260}

Submitted on 1 Jan 1981

HAL is a multi-disciplinary open access archive for the deposit and dissemination of scientific research documents, whether they are published or not. The documents may come from teaching and research institutions in France or abroad, or from public or private research centers.
L'archive ouverte pluridisciplinaire HAL, est destinée au dépôt et à la diffusion de documents scientifiques de niveau recherche, publiés ou non, émanant des établissements d'enseignement et de recherche français ou étrangers, des laboratoires publics ou privés. 


\title{
Étude de quelques facteurs de variation de la fécondité chez Trichogramma maidis Pintureau et Voegele (Hym. Trichogrammatidae)
}

\author{
Bernard PINTUREAU, Manuel BABAULT \& Jean VOEGELE \\ I.N.R.A., Station de Zoologie et de lutte biologique, 37, bd du Cap, F 06602 Antibes.
}

\author{
RÉSUMÉ \\ Insecte, \\ Hyménoptère, \\ Trichogrammatidae, \\ Trichogramma maidis, \\ Fécondité \\ Variabilité génétique, \\ Variabilité épigénétique.
}

Chez une souche de Trichogramma muidis, en élevage prolongé, la fécondité présente une variabilité génétique extrêmement faible que nous n'avons pas pu déceler. La longévité variable et la fécondation ou non des femelles influent par contre beaucoup sur ce caractère. Pour comparer les populations, une mesure de la fécondité éliminant le maximum de variabilité épigénétique est choisie : 7 premiers jours de ponte, femelles fécondées, conditions climatiques contrôlées $\left(25^{\circ} \mathrm{C}, 70 \mathrm{p} .100 \mathrm{H} . \mathrm{R} ., 16 \mathrm{~h}\right.$ d'éclairement par jour).

\section{SUMMARY \\ Insect, \\ Hymenoptera, \\ Trichogrammatidae, \\ Trichogramma maidis, \\ Fecundity, \\ Genetic variability, \\ Epigenetic variability.}

Study of some factors of variation of the fecundity in Trichogramma maidis Pintureau \& Voegele (Hym. Trichogrammatidae).

In a strain of $T$. maidis in lengthened rearing, the fecundity shows an extremely low genetic variability that we cannot reveal. On the other hand the variable longevity and the fecondation or not of females influence on this character. To compare the populations, a measure of the fecundity excluding the most part of epigenetic variability is chosen : 7 first days of egg-laying, fecundated females, climatic conditions controlled $\left(25^{\circ} \mathrm{C}\right.$, 70 p. 100 R.H., 16 h of light a day).

\section{INTRODUCTION}

La mesure de la fécondité (nombre d'œufs pondus) est longue et laborieuse chez les trichogrammes comme chez tous les parasites oophages d'insectes. C'est pourquoi, très souvent, d'autres caractères pouvant indirectement apprécier la fécondité sont mesurés :

- Nombre d'ovocytes contenus dans les ovarioles (VOEGELE, 1969) ;

- Nombre de descendants adultes, appelé parfois productivité (WATANABE \& WATANABE, 1973; BIEMONT, 1978 ; BIEMONT \& BOULETREAU, 1980), viabilité (BIEMONT, 1978), taux de multiplication ou fertilité (VOEGELE et al., 1975)

- Nombre d'œufs-hôtes parasités (facilement reconnaissables, dans le cas de trichogrammes, à leur teinte foncée), en connaissant le nombre moyen de parasites par hôte, appelé également parfois productivité (LUND, 1938).

Notons que le terme de fertilité, au sens de nombre d'œufs fécondés, ne signifie rien chez les Hyménoptères, sinon pour exprimer le futur taux sexuel. Il est en revanche parfois utilisé au sens de nombre d'œufs se développant (donnant des larves) ou même de pourcentage d'éclosion
(HOLLINGSWORTH \& MAYNARD-SMITH, 1955 ; DAVID et al., 1975).

Chez les trichogrammes, insectes utilisés en lutte biologique, ces caractères que nous nommerons globalement fécondité font souvent l'objet de comparaisons entre espèces ou populations. La variabilité intra-population, qui peut avoir une origine génétique et épigénétique, est importante et il est utile de rechercher plus précisément ses causes.

En ce qui concerne la variabilité épigénétique, de nombreux facteurs, dont nous donnons une liste non exhaustive, peuvent intervenir :

\section{Facteurs abiotiques}

- Climat : température, humidité, photopériode, vents, etc. (LUND, 1938).

\section{Facteurs biotiques}

-w Nutrition: quantité et qualité de la nourriture de l'imago (LUND, 1938 ; ASHLEY \& GONZALEZ, 1974), quantité de nourriture de la larve en rapport avec la taille des 
œufs-hôtes et le superparasitisme (LUND, 1938). Une larve bien nourrie donnera une femelle plus grosse et plus féconde.

- Nature des æufs-hôtes et quantité : une femelle pond plus ou moins en fonction de l'espèce-hôte et de son abondance (FLANDERS, 1935 ; NGUYEN BAN, 1974), mais aussi en fonction de l'âge des embryons (BENOIT \& VOEGELE, 1979). En cas d'absence temporaire d'hôte, la rétention des œufs par le parasitoïde exerce une influence sur sa fécondité (LUND, 1938).

- Effets maternels et effets prolongés (étudiés en particulier chez les drosophiles par DAvID, 1961) : le vieillissement de la mère conduit à une diminution de la fécondité des filles (BENOIT, 1977), le fait que la mère a ou non diapausé exerce aussi une influence.

- Effets de groupe et de masse (KUNO, 1962).

\section{Facteurs expérimentaux}

- Préparation des hôtes de substitution : durée d'exposition des cufs aux U.V., durée de stockage au froid (VOEGELE et al., 1975).

- Volume d'élevage.

- Erreurs de mesure.

Autres caractères (pouvant également être soumis à des facteurs épigénétiques)

- Longévité des femelles : cet autre caractère est corrélé avec la fécondité (LUND, 1938).

- Fécondation ou non des femelles (LUND, 1938), nombre de fécondations.

A la variation due aux causes connues s'en ajoute une autre, dite intangible (FALCONER, 1974) et d'origine indéterminée (on peut, par exemple, penser aux facteurs abiotiques mal étudiés tels que pression atmosphérique, champs magnétique et électrique, radiations cosmiques, etc...).

Après l'étude de plusieurs facteurs de variabilité, nous essayerons de choisir une mesure de fécondité éliminant au maximum la variation épigénétique et donnant plus de signification aux comparaisons de populations.

\section{MATÉRIEL ET MÉTHODES}

Une souche de Trichogramma maidis PINTUREAU \& VOEGELE (numérotée 16 dans nos élevages) a fourni le matériel d'étude. Un faible nombre d'individus ( 1 q et 3 ơ) ont fondé la souche, arrivée en novembre 1973 au laboratoire. Elle a, depuis, été maintenue en élevage sur œufs d'Ephestia kuehniella Zeller (Lep. Pyralidae).

Les individus sont originaires d'une population de Moldavie (U.R.S.S.) qui parasite surtout les cufs d'Ostrinia nubilalis Hübner (Lep. Pyralidae).

Nous avons étudié, comme critères quantitatifs, soit le nombre de descendants adultes (fécondité moins mortalité embryonnaire et larvo-nymphale), soit le nombre d'œufs d'E. kuehniella parasités. Ce dernier critère, en tenant compte qu'il n'y a, en principe qu'un parasite par hôte lorsque ceux-ci sont excédentaires, correspond en fait au nombre de prénymphes ou de nymphes du parasite (fécondité moins mortalité embryonnaire et des premiers stades larvaires). Ces 2 mesures (fig. 1) sont fortement corrélées : $p=0,958$ pour $n=116$, ceci pour les 7 premiers jours de ponte des femelles fécondées, dans des conditions climati-

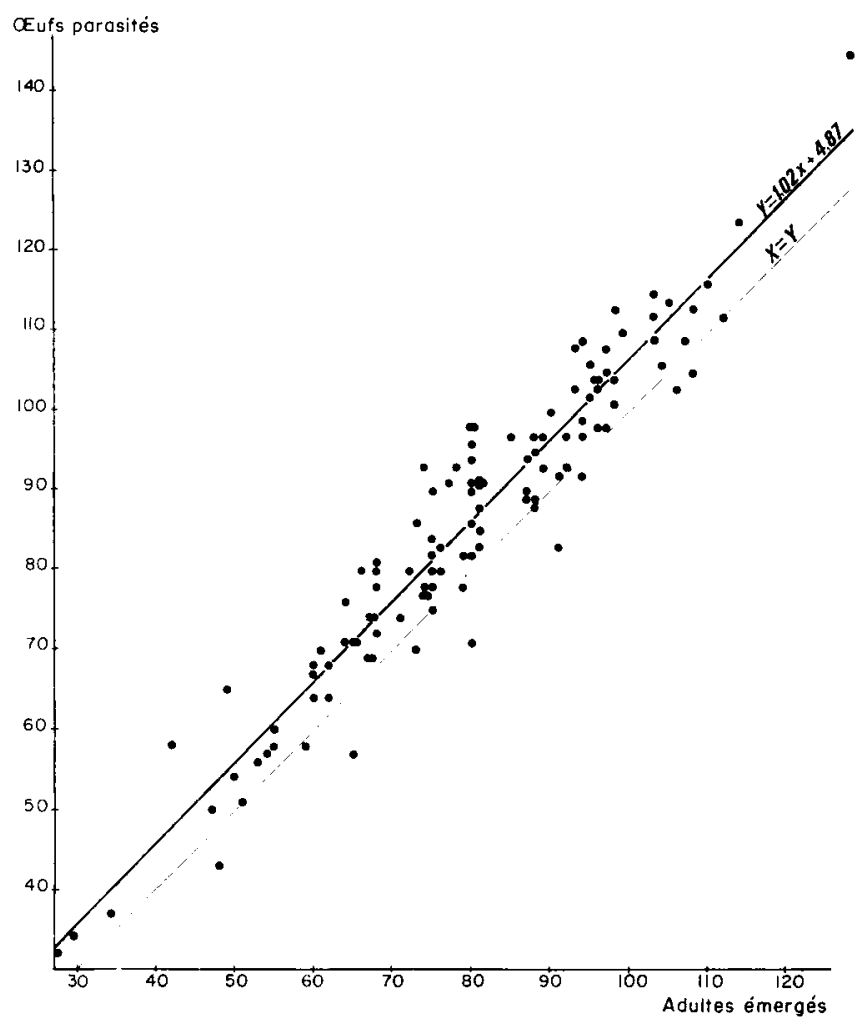

Figure 1

Régression ( $y=1,02 x+4,87)$ des aufs parasités par une femelle pendant 7 jours sur les adultes émergés de ces mêmes oufs. En principe, le nombre d'adultes est inférieur au nombre d'aufs parasités, toutefois dans quelques cas le superparasitisme compense et dépasse les mortalités nymphales (points situés à droite de la ligne pointillée).

Regression ( $y=1,02 x+4,87)$ of parasitized eggs by a female during 7 days on emerged adults from the same eggs. Fundamentally, the number of adults is lesser than the number of parasitized eggs, but sometimes the superparasitism balances and exceeds the nymphal mortalities (points situated on the right of the dotted line).

ques contrôlées $\left(25^{\circ} \mathrm{C}, 70\right.$ p. $100 \mathrm{H}$.R. et 16 h d'éclairement par jour). Les trichogrammes seront toujours soumis à ces conditions de température d'humidité et de photopériode, sauf indication contraire.

Nous prendrons donc pour évaluer la fécondité, indifféremment mais en le précisant, les dénombrements d'œufs parasités ou d'adultes émergés.

Dans un premier temps, nous étudierons la variabilité génétique au moyen du calcul de l'héritabilité. La méthode utilisée est celle de la régression des filles sur la mère. Nous mesurerons ensuite l'influence de 4 facteurs épigénétiques ou caractères liés à la fécondité en n'en faisant varier qu'un seul à la fois : durée de ponte en rapport avec la longévité, fécondation, effet maternel et conditions climatiques. L'effet maternel étudié est celui mis en évidence par BENOIT (1977) : le vieillissement de la mère exerce une action sur la fécondité (nombre d'œufs d'E. kuehniella parasités) des filles; les trichogrammes femelles issus d'œufs pondus tôt par la mère pondent plus. Nous comparerons les variances par le test $F$ et les moyennes par le test $t$ Afin d'apprécier les variabilités, sans qu'un effet d'échelle intervienne, nous avons aussi calculé les coefficients de variation ; C.V. $=100 \frac{\sigma}{\bar{x}}$ (DAvID et al., 1978).

Dans tous nos calculs, nous ne tiendrons pas compte des femelles stériles car cela reviendrait à mélanger 2 types d'individus (stérile-fécond) et donnerait à l'ensemble une distribution s'éloignant de la normale. 


\section{RÉSULTATS}

\section{Variabilité génétique}

L'héritabilité a été calculée à partir du nombre d'œufs d'E. kuehniella parasités par des femelles fécondées. Les couples sont nourris de miel et disposent d'un nombre d'hôtes constant, en excès par rapport à leurs besoins.

En tenant d'abord compte de la fécondité totale (de toute la vie des femelles), nous avons répété les calculs sur 2 lots de filles : l'un comprend toutes les filles, l'autre seulement celles issues du $1^{\text {er }}$ quart d'heure de ponte de la mère. Nous avons voulu supprimer, en $2^{\mathrm{e}}$ analyse, l'effet maternel lié au vieillissement, les mères étant elles-mêmes issues des 3 premières heures de ponte. Ces calculs ont été effectués au bout de 3 ans et demi d'élevage.

Dans les 2 cas (tabl. 1, fig. 2A et $2 \mathrm{~B}$ ), l'héritabilité $\left({ }^{1}\right)$ est nulle en tenant compte de l'erreur. Nous remarquons dans le tableau 1 que la fécondité des mères est supérieure à celle des filles, cette différence est probablement due à un léger tri des mères (petites, peu actives) tandis que le choix était plus réduit pour les filles. Aucune ressemblance entre apparentés, même épigénétique, n'est décelée.

Nous avons effectué un $3^{\mathrm{e}}$ calcul à partir de la fécondité des 7 premiers jours de ponte afin d'éliminer l'influence de longévité. Ici, nous n'avons pas tenu compte de l'effet maternel (mères et filles prises au hasard) et le nombre d'individus analysés est plus grand (47 mères au lieu de 17). Ce calcul a été effectué au bout de 6 ans d'élevage. Nous obtenons de nouveau (tabl. 1, fig. 2C) une héritabilité nulle.

Ces résultats montrent que la variabilité génétique doit être extrêmement faible et que nous allons pouvoir étudier l'influence du milieu (facteurs épigénétiques) ou d'autres caractères sur la fécondité sans nous en soucier.

Le calcul du coefficient de consanguinité théorique est d'ailleurs en accord avec le calcul de l'héritabilité :

La $1^{\text {re }}$ génération d'élevage, issue de 1 q et $3 \delta$ supposés non apparentés, donne un coefficient nul.

La $2^{\mathrm{e}}$ génération, qui est composée de la descendance de croisements frères-sœurs (ce qui équivaut à une parenté mère-fille chez les diplo-diploïdes), donne un coefficient égal à 0,25 (FALCONER, 1974).

Le coefficient de consanguinité peut ensuite être calculé à l'aide de la formule $F_{t}=\Delta F+(1-\Delta F) F_{t-1}$, où $t$ représente la génération et $\Delta \mathrm{F}$ l'accroissement de consanguinité en une génération proportionnellement à la distance qu'il reste à parcourir pour atteindre la consanguinité absolue.

$\Delta \mathrm{F}$, qui est égal à $\frac{1}{8 \mathrm{Nm}}+\frac{1}{8 n f}$ chez les diplo-diplö̈des (Wright, 1931), devient $\frac{1}{9 \mathrm{Nm}}+\frac{2}{9 \mathrm{~N} f}$ chez les haplodiploïdes (Wright, 1933) où $\mathrm{N} m$ et $\mathrm{N} f$ représentent respectivement le nombre de mâles et de femelles contribuant à la génération suivante (effectif efficace).

Les repiquages ayant été effectués à l'aide de 50 individus environ $(31 q$ et 19 o car les femelles représentent 63 p. 100 en moyenne), $\Delta \mathrm{F}$ est ici égal à 0,013 .

Ainsi, $F_{t}$ est égal à 0,260 à la $3^{\text {e }}$ génération et à 0,270 à la 4 e. L'augmentation étant de 0,010 par génération, la consanguinité devient théoriquement totale à la $77^{\mathrm{e}}$ génération ( 3 ans d'élevage environ, une génération durant 14 jours en

(1) Si $b$ est la régression des filles sur la mère, $h^{2}=2 b$ et $\sigma^{2} h^{2}=4 \sigma_{b}^{2}$, la variance de $b$ est $\frac{1}{n-2}\left(\frac{\sigma^{2} \text { mères }}{\sigma^{2} \text { filles }}-b^{2}\right)$.

(Falconer, 1974).
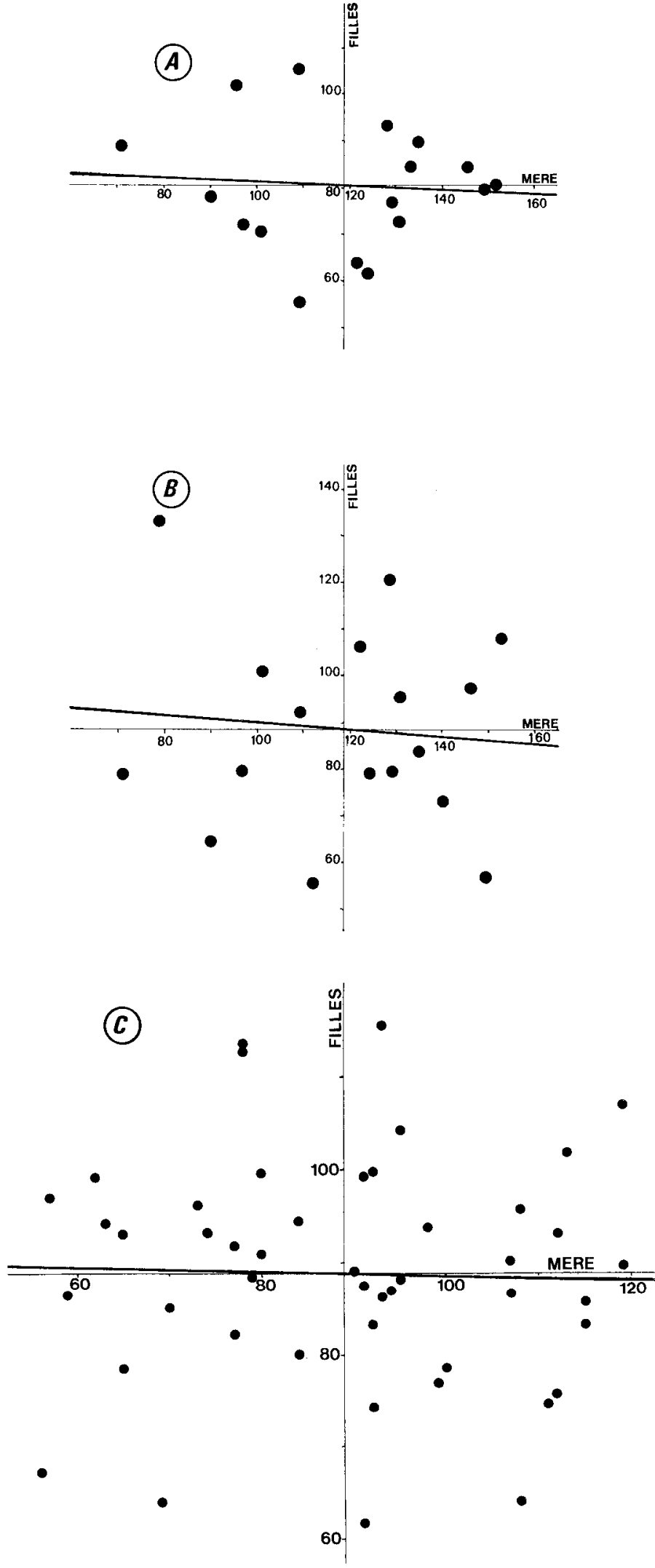

Figure 2

Régression des filles sur la mère pour la fécondité (nombre d'aufs hôtes renfermant des nymphes de parasite) totale ( $A$ et $B$ ) ou des 7 premiers jours de ponte $(C)$. En $A$, nous avons tenu compte de toutes les filles et en $B$, seulement de celles issues du $1^{\text {er }}$ quart d'heure de ponte.

Regression of daughters on the mother for total fecundity (number of host-eggs containing parasite-nymphs) ( $A$ and $B$ ) or for the fecundity during the first 7 days of egglaying $(C)$. In $A$ we consider all the daughters and in $B$ those who were born in the first quarter of their mother's egglaying. 


\section{TABLEAU 1}

Calcul de l'héritabilité de la fécondité (nombre d'cufs-hôtes renfermant des nymphes du parasite) de toute la vie et des 7 premiers jours de ponte. Dans le premier cas, nous avons tenu compte de toutes les filles dont nous avons disposé, puis seulement des filles issues du premier quart d'heure de ponte ( $n$ : effectif ; $\bar{x}$ et $\bar{y}:$ moyennes ; $\sigma$ écart type ; $\sigma^{2}$ : variance)

Calculation of the heritability of the fecundity (number of host-eggs containing parasite-nymphs) of the whole life and of the first 7 days of egglaying. In the first case, we consider all the daughters we obtained, then the daughters who were born in the first quarter of egglaying ( $n$ : sample size ; $\bar{x}$ and $\bar{y}$ : means; $\sigma$ : standard deviation ; $\sigma^{2}$ : variance)

\begin{tabular}{|c|c|c|c|}
\hline \multicolumn{4}{|c|}{ Fécondité totale } \\
\hline & Ensemble des filles & $\begin{array}{c}\text { Filles issues du premier } 1 / 4 \mathrm{~h} \\
\text { de ponte }\end{array}$ & Fécondité des 7 premiers jours \\
\hline $\mathrm{n}$ mères & 17 & 17 & 47 \\
\hline $\mathrm{n}$ de filles/mère & 3 à $8(\bar{x}=6,2)$ & 2 à $4(\bar{x}=2,4)$ & 4 à $10(\bar{x}=5,9)$ \\
\hline$\overline{\mathbf{x}}$ mères & $119,0 \pm 5,5$ & $118,5 \pm 5,9$ & $89,0 \pm 2,6$ \\
\hline$\sigma x$ & 22,6 & 24,4 & 17,9 \\
\hline $\bar{y}$ filles & $80,2 \pm 3,3$ & $88,5 \pm 5,2$ & $89,0 \pm 1,8$ \\
\hline oy & 13,5 & 21,3 & 12,3 \\
\hline$b=$ coeff. de regression & \multirow{2}{*}{$-0,04 \pm 0,10$} & $-0,07 \pm 0,07$ & $-0,02 \pm 0,03$ \\
\hline$\sigma^{2}$ & & $0,0 \overline{9}$ & 0,05 \\
\hline Droite de regression & $y=-0,04 x+84,93$ & $y=-0,07 x+96,27$ & $y=-0,02 x+90,61$ \\
\hline $\mathrm{h}^{2}=$ héritabilité & $-0,08 \pm 0,21$ & $-0,13 \pm 0,14$ & $-0,04 \pm 0,06$ \\
\hline$\sigma_{\mathrm{h} 2}$ & 0,75 & 0,35 & 0,19 \\
\hline
\end{tabular}

moyenne aux températures du laboratoire qui varient entre 20 et $25^{\circ} \mathrm{C}$ ).

Ce calcul théorique est peut-être surestimé car il ne peut tenir compte des nombreux mécanismes de maintien du polymorphisme (mutations, sélection variable, hétérosis, etc.).

En tout état de cause, la consanguinité n'entraîne pas, chez les trichogrammes, une dépression sur la fécondité (nombre de descendants adultes) comparable à celle observée chez Drosophila subobscura Collin (Dipt. Drosophilidae) par HOLLINGSWORTH \& MAYNARD-SMITH, 1955. La situation est plus proche de celles constatées par HELLE (1965) chez Tetranychus urticae Koch (Acarien Tetranychidae) et BIEMONT \& BOULETREAU (1980) chez Cothonaspis boulardi Barbotin, Carton \& Kelner-Pillault (Hym. Eucoilidae).

\section{Variabilité épigénétique ou due à d'autres caractères}

Nous n'allons analyser l'influence que de quelques sources de variabilité sur la fécondité et relever leur importance : longévité, fécondation, effet maternel en rapport avec l'âge de la mère et climat.

En conséquence, les autres facteurs seront constants dans toutes nos expériences : imagos nourris de miel; pas d'effets de groupe, de masse ou en rapport avec la diapause ; œufs-hôtes (E. kuehniella) en quantité suffisante limitant au maximum le superparasitisme, d'âge constant (1 j) et ayant subi la même dose d'ultra-violets (à $25 \mathrm{~cm}$ de 4 lampes de $25 \mathrm{~W}$ pendant $20 \mathrm{~min}$ ) et le même stockage (à $3{ }^{\circ} \mathrm{C}$ pendant 1 semaine au plus) ; limitation de la rétention des œufs par les parasitoïdes (pas d'absence d'hôte); volume d'élevage constant. Nous considérons les erreurs de mesure comme négligeables.

\section{a) Influence de la longévité des femelles et du rythme de changement des hôtes sur la fécondité}

Nous avons comparé la fécondité (nombre d'œufs-hôtes parasités) d'un lot de femelles qui a pondu durant toute sa vie (10 à $11 \mathrm{j}$ en moyenne) et d'un lot qui a pondu durant $7 \mathrm{j}$.

Les mesures sont faites sur des femelles fécondées (couples isolés), l'effet maternel s'applique également sur les 2 lots.

Les trichogrammes exercent leur activité parasitaire plus intensément pendant les premiers jours de leur vie et, au bout d'une semaine, la majeure partie de leur ponte est effectuée ; la différence entre les 2 lots n'en demeure pas moins significative (tabl. 2).

\section{TABLEAU 2}

Fécondités (oufs-hôtes parasités), de femelles fécondées, pendant les 7 premiers jours de ponte et pendant toute la vie. Comparaison des variances $\left(\sigma^{2}\right)$ et des moyennes $(\bar{x})$ par les tests $F$ et $t(n$ : effectif ; C.V. : coefficient de variation; ** : seuil à 1 p. 100) Fecundities (parasitized host-eggs), of mated females, during the first 7 days of egglaying and during the whole life. Comparison of variances $\left(\sigma^{2}\right)$ and means $(\bar{x})$ by $F$ and t tests $(n:$ sample size $; C . V$. : coefficient of variation; ${ }^{* *}:$ threshold 1 p. 100)

\begin{tabular}{|c|c|c|c|c|c|c|}
\hline & $\mathrm{n}$ & $\bar{x}$ & $\sigma^{2}$ & C.V. & $F$ & $t$ \\
\hline 7 premiers jours & 15 & $63,3 \pm 2,8$ & 119,0 & 17,2 & 8,36 & 4,24 \\
\hline Toute la vie & 121 & $80,6 \pm 3,0$ & 1057,1 & 40,3 & $* *$ & ** \\
\hline
\end{tabular}

Nous constatons surtout que les différences de longévité entraînent une variabilité de la fécondité très importante. La comparaison des variances donne un coefficient $F$ hautement significatif, les coefficients de variations passent du simple à plus du double.

De manière à connaître la fécondité partielle minimum ayant une bonne corrélation avec la fécondité totale, nous avons effectué 2 expérimentations (les mesures sont les mêmes que précédemment et prises dans les mêmes conditions) :

- des femelles isolées (10 répétitions) ont été mises en présence d'œufs-hôtes renouvelés tous les jours. Nous avons ainsi obtenu la fécondité correspondant au $1^{\text {er }}$ jour de ponte et, en cumulant, aux 2, 3, etc., premiers jours de ponte, pour finalement obtenir la mesure totale. Les corrélations (fig. 3) existant entre les diverses fécondités partielles, jusqu'à $14 \mathrm{j}$ compris, et la fécondité totale ont été 
calculées; nous constatons que les 7 premiers jours de ponte donnent un résultat très bien corrélé $(p=0,90)$ avec celui de l'ensemble de la ponte ;

- la $2^{\mathrm{e}}$ expérience a permis de contrôler si le fait de changer les hôtes tous les jours avait une influence sur la fécondité. 6 séries de mesures (10 répétitions pour chacune) ont été faites en changeant les hôtes tous les $2 \mathrm{j}, 3 \mathrm{j}$, etc..., jusqu'à $7 \mathrm{j}$; dans chaque cas le cumul donne la fécondité

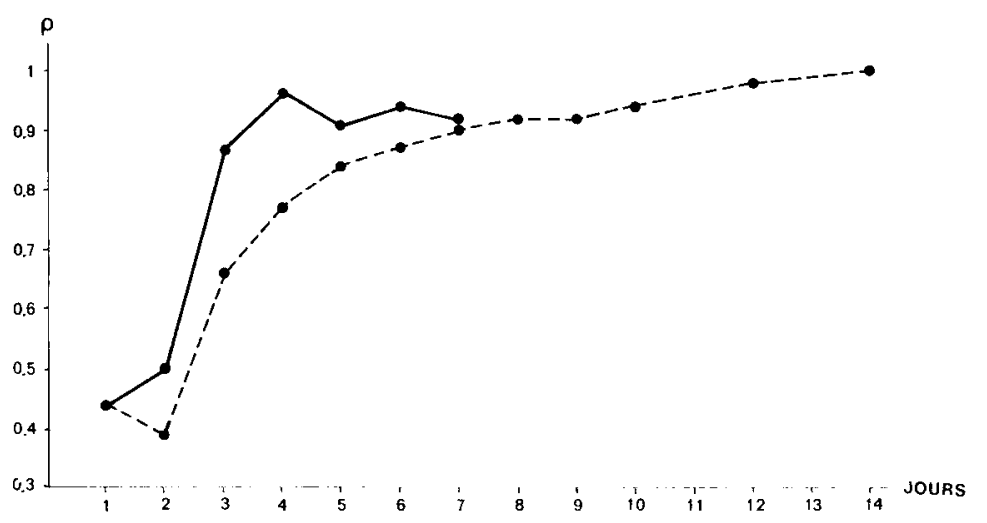

totale. Nous avons constaté qu'il n'y a pas de différence significative (test $t$ ) entre les fécondités moyennes selon que l'on renouvelle ou non les hôtes, la « fraîcheur » des oufs d'Ephestia (tués aux ultra-violets et stockés à $3{ }^{\circ} \mathrm{C}$ ) n'a donc pas d'influence décelable dans la limite de $7 \mathrm{j}$. Le calcul des corrélations (fig. 3) entre fécondités partielle et totale montre que le coefficient est élevé pour des périodes inférieures à celles de l'expérience précédente.

Figure 3

Coefficients de corrélation entre la fécondité totale et les fécondités partielles correspondant au premier, deux premiers, etc., jours de ponte.

Trait plein : les aufs-hôtes ne sont pas renouvelés durant la période de mesure.

Tirets : les oufs-hôtes sont renouvelés tous les jours.

Coefficients of correlation between the total fecundity and the partial fecundities for the first two, etc., days of egglaying.

Full line: the host-eggs are not renewed during the period of measure.

Dashes : the host-eggs are renewed every day.

\section{b) Influence de la fécondation des femelles surleur fécondité}

Nous avons comparé la fécondité (nombre d'adultes émergeant) correspondant aux 3,5 et 7 premiers jours de ponte de femelles fécondées ou vierges, sans tenir compte du vieillissement maternel.

Contrairement à ce qui a été observé chez les Drosophiles (COHET \& BOUlETREAU-MERLE, 1974), les femelles vierges de trichogrammes pondent plus que les femelles fécondées (phénomène déjà observé par LUND, 1938), la différence étant significative pour les périodes de ponte de 3 et $7 j$ (tabl. 3). En ce qui concerne la période de $5 \mathrm{j}$, le résultat est différent (fig. 4), les femelles vierges pondent en effet au bout de cette période sensiblement autant qu'en $3 \mathrm{j}$.

Ceci nous amène à penser qu'un cycle d'ovogenèse se produit, avec une période de repos entre le $3^{\mathrm{c}}$ et le $5^{\mathrm{e}} \mathrm{j}$, phénomène qui serait en partie estompé par la fécondation. Des dissections permettraient d'apporter une réponse définitive à ces suppositions, cependant l'analyse des variances appuie cette interprétation : la variance est plus forte pour les fécondités correspondant aux 5 premiers jours de ponte, à ce moment des femelles auraient commencé leur $2^{\mathrm{e}}$ cycle et d'autres pas.

Bien que la différence entre les variances des fécondités de femelles vierges et fécondées ne soit significative que pour la période de $3 \mathrm{j}$, il semble qu'elle soit réelle : la variance est plus faible lorsque la mesure concerne les femelles fécondées et le rôle régulateur de la fécondation apparaît de nouveau. Les coefficients de variation (tabl. 3) sont les plus différents pour la période de $5 \mathrm{j}$.

La régularisation de la ponte pourrait s'expliquer par le fait que, chez les femelles fécondées, une partie des cufs doit recevoir des gamètes mâles au passage devant l'orifice de la spermathèque ; les femelles vierges, quant à elles, pondraient plus vite et moins régulièrement. GERBER \& KLOSTERMEYER (1970) ont ainsi constaté un tel phénomène chez Megachile rotundata F. (Hym. Apidae) : les femelles observent une pause avant de pondre un œuf fécondé, pause qui n'existe pas pour un ouf non fécondé ; les auteurs pensent que le phénomène correspond au positionnement interne de l'œuf pour recevoir le sperme.

\section{c) Influence du vieillissement de la mère sur la fécondité des filles}

Cet effet maternel provient probablement d'une variation de la qualité du contenu ovulaire au cours de la vie maternelle dont les conséquences persistent chez l'adulte.

\section{TABLEAU 3}

Fécondités (adultes émergeant), de femelles fécondées et vierges, pendant les 3, 5 et 7 premiers jours de ponte. Comparaison des variances ( $\left.\sigma^{2}\right)$ et des moyennes $(\vec{x})$ par les tests $F$ et $t$ ( $n:$ effectif ; C.V. : coefficient de variation ; $*$ seuil à 5 p. $100 ; * *:$ seuil à 1 p. 100 )

Fecundities (emerging adults) of mated and virgin females, during the first 3,5 and 7 days of egglaying. Comparison of variances ( $\sigma^{2}$ ) and means $(\bar{x})$ by $F$ and $t$ tests ( $n$ : sample size ; C.V. : coefficient of variation; * threshold 5 p. $100 ; * *:$ threshold 1 p. 100)

\begin{tabular}{|c|c|c|c|c|c|c|c|}
\hline \multirow{3}{*}{3 premiers jours } & & $\mathrm{n}$ & $\overline{\mathrm{X}}$ & $\sigma^{2}$ & C.V. & $\mathrm{F}$ & $\mathrm{t}$ \\
\hline & & 15 & $33,5 \pm 2,0$ & 60,9 & 23,3 & \multirow{2}{*}{$\begin{array}{c}2,64 \\
*\end{array}$} & \multirow{2}{*}{$\begin{array}{c}3,37 \\
* * *\end{array}$} \\
\hline & Femelles vierges & 15 & $46,5 \pm 3,3$ & 160,8 & 27,3 & & \\
\hline \multirow[b]{2}{*}{5 premiers jours } & Femelles fécondées & 15 & $44,7 \pm 2,6$ & 100,2 & 22,4 & \multirow[b]{2}{*}{2,08} & \multirow[b]{2}{*}{0,42} \\
\hline & Femelles vierges & 16 & $46,6 \pm 3,6$ & 209,9 & 31,1 & & \\
\hline \multirow{2}{*}{7 premiers jours } & Femelles fécondées & 15 & $51,9 \pm 2,3$ & 80,2 & 17,3 & \multirow{2}{*}{2,35} & \multirow{2}{*}{$\underset{*}{2,28}$} \\
\hline & Femelles vierges & 15 & $61,5 \pm 3,5$ & 188,2 & 22,3 & & \\
\hline
\end{tabular}



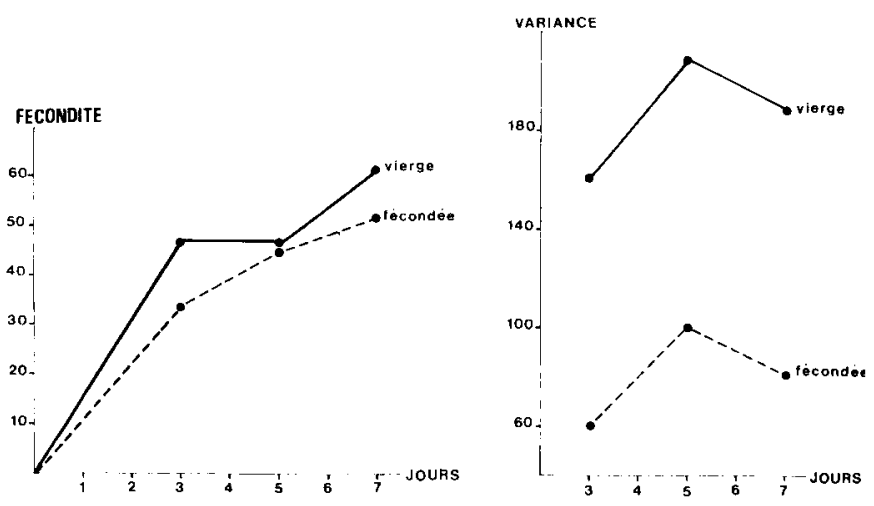

Afin de connaître l'influence de ce facteur sur la mesure de la fécondité, nous avons comparé les fécondités totales (nombre d'œufs parasités) de 2 lots de femelles fécondées. Un $1^{\text {er }}$ est homogène en ce qui concerne l'effet maternel (toutes les femelles sont issues du $1^{\text {er }}$ quart d'heure de ponte de la mère), un $2^{\mathrm{e}}$ est hétérogène quant à cet effet (les femelles sont issues du $1^{\text {er }}$ quart d'heure, des $3^{\mathrm{e}}, 5^{\mathrm{e}}$ et $7^{\mathrm{e}} \mathrm{j}$ de ponte).

Le tableau 4 montre que les moyennes et variances des 2 lots de femelles sont statistiquement identiques; les coefficients de variations ne diffèrent pas beaucoup. Bien que l'influence de l'effet maternel soit ici maximum, puisque dans un lot de femelles toutes les filles sont prises en considération, elle n'agit pas sur les moyennes des fécondités totales qui peuvent donc être calculées à partir d'individus pris au hasard. L'effet maternel n'est donc sensible qu'entre 2 lots de femelles ayant subi des effets maternels différents mais précis. Selon les données de BENOIT (1977), la différence d'après le test « $t$ » n'est hautement significative (seuil 1 p. 100) qu'entre les lots de filles jssues respectivement du $1^{\text {er }}$ quart d'heure et du $7^{\mathrm{e}} \mathrm{j}$ de ponte de leurs mères ; elle est significative (seuil 5 p. 100) entre lots de filles issues du $3^{\mathrm{e}}$ et $\mathrm{du} 7^{\mathrm{e}} \mathrm{j}$ de ponte de leurs mères. II faut noter que l'influence de l'effet maternel doit mieux apparaître dans le cas des fécondités de $7 \mathrm{j}$ dont la variance est bien moindre.

\section{TABLEAU 4}

Fécondités (aufs-hôtes parasités) totales de femelles fécondées issues du premier quart d'heure de ponte de leur mère et de l'ensemble de la ponte de leur mère. Comparaison des variances $\left(\sigma^{2}\right)$ et des moyennes $(\bar{x})$ par les tests $F$ et $t$ ( $n$ : effectif ; C.V. : coefficient de variation)

Total fecundities (parasitized host-eggs) of mated females who were born in the first quarter of the egglaying of their mother and in all the egglaying of their mother. Comparison of variances $\left(\sigma^{2}\right)$ and means $(\bar{x})$ by $F$ and t tests ( $n$ : sample size ; C.V. : coefficient of variation)

\begin{tabular}{|c|c|c|c|c|c|c|}
\hline & $\mathbf{n}$ & $\overline{\mathrm{x}}$ & $\sigma^{2}$ & C.V. & $\mathrm{F}$ & $\mathrm{t}$ \\
\hline $\begin{array}{l}\text { Filles issues du } \\
\text { premier } 1 / 4 \mathrm{~h} \text { de } \\
\text { ponte }\end{array}$ & 44 & $88,4 \pm 4,7$ & 967,6 & 35,2 & 1,08 & 1,41 \\
\hline $\begin{array}{l}\text { Ensemble des } \\
\text { filles }\end{array}$ & 121 & $80,6 \pm 3,0$ & 1057,1 & 40,3 & & \\
\hline
\end{tabular}

\section{d) Influence des conditions climatiques sur la fécondité}

Nous avons mesuré la fécondité (nombre d'adultes émergeant) des 3 premiers jours de ponte de femelles fécondées soumises soit aux conditions climatiques stables précédentes soit aux conditions variables $\left(20\right.$ à $25^{\circ} \mathrm{C}, 60$ à 80 p. 100 H.R., photopériode naturelle) du laboratoire entre les mois

\section{Figure 4}

Fécondité et sa variance mesurées pendant les 3,5 et 7 premiers jours de ponte chez les femelles vierges et fécondées.

Measured fecundity and its variance during the first 3,5 and 7 days of egglaying for virgin and mated females.

d'avril et de novembre. $T$. maidis présente, en conditions constantes, un optimum thermique de $23^{\circ} \mathrm{C}$ pour le développement et de $25^{\circ} \mathrm{C}$ pour la ponte (RUsso, 1980).

Les variations climatiques (tabl. 5) conduisent à une fécondité supérieure tandis qu'elles n'ont pas d'influence significative sur la variance. Ces conditions fluctuantes sont insuffisantes pour augmenter la variabilité de la fécondité mais des conditions plus extrêmes aboutiraient probablement à un tel résultat.

\section{TABLEAU 5}

Fécondités (adultes émergeants), de femelles fécondées, pendant les 3 premiers jours de ponte dans des conditions climatiques stables et fluctuantes. Comparaison des variances $\left(\sigma^{2}\right)$ et des moyennes $(x)$ par les tests $F$ et $t$ ( $n$ : effectif ; $C$. V. : coefficient de variation ; ${ }^{* *}:$ seuil à 1 p. 100)

Fecundities (emerging adults), of mated females, during the first 3 days of egglaying under stable and fluctuating climatic conditions. Comparison of variances $\left(\sigma^{2}\right)$ and means $(\bar{x})$ by $F$ and $t$ tests $(n$ : sample size ; C.V. : coefficient of variation ; ${ }^{* *}:$ threshold 1 p. 100)

\begin{tabular}{lcccccc}
\hline \hline & $\mathrm{n}$ & $\overline{\mathrm{x}}$ & $\sigma^{2}$ & $\mathrm{C} . \mathrm{V}$. & $\mathrm{F}$ & $\mathrm{t}$ \\
$\begin{array}{l}\text { Conditions cli- } \\
\text { matiques stables }\end{array}$ & 15 & $33,5 \pm 2,0$ & 60,9 & 23,3 & 1,76 & 2,82 \\
$\begin{array}{l}\text { Conditions cli- } \\
\text { matiques fluc- } \\
\text { tuantes }\end{array}$ & 70 & $40,2 \pm 1,3$ & 113,3 & 26,4 & & $* *$ \\
\hline
\end{tabular}

\section{DISCUSSION ET CONCLUSION}

La fécondité des trichogrammes, appréciée au moyen du nombre d'œufs d'E. kuehniella parasités ou du nombre d'imagos fils ( 2 mesures en très forte corrélation), est sensible à de nombreux facteurs. Chez la souche étudiée, la variation génétique semble extrêmement faible, par contre des facteurs tels que la longévité et la fécondation ou non des femelles jouent un rôle important. Notons que ces caractères agissent peut-être à la fois sur la fécondité et sur le taux de mortalité embryonnaire et des premiers stades larvaires sans que nous puissions l'appréhender.

Les différences de longévité des femelles conduisent à une grande variabilité de la fécondité ; les 7 premiers jours de ponte rendent cependant compte de la plus grande partie de l'activité reproductrice.

Les femelles vierges pondent plus que les femelles fécondées et il semble que la fécondation ait un rôle régularisateur sur la ponte.

La fluctuation des conditions climatiques, avec une amplitude limitée $\left(20\right.$ à $25^{\circ} \mathrm{C}, 60$ à 80 p. 100 H.R., photopériode naturelle), augmente la fécondité sans affecter sa variabilité. Enfin l'effet maternel dû au vieillissement de la mère a une influence plus réduite sur le caractère étudié. 
La fécondité, qui fait fréquemment l'objet de comparaisons entre populations de trichogrammes, doit être mesurée avec le moins possible de variabilité épigénétique. La mesure qui est effectuée sur les 7 premiers jours de ponte (élimination du facteur longévité) de femelles fécondées (variance inférieure) dans des conditions climatiques contrôlées (par exemple $25^{\circ} \mathrm{C}, 70$ p. 100 H.R., 16 h d'éclairement par jour) paraît ainsi convenable. Il est bon également d'éliminer les femelles stériles, ces individus pouvant perturber la loi normale et les comparaisons entre populations.

Précisons que la ponte des 7 premiers jours qui est bien corrélée avec la ponte totale chez $T$. maidis ne le sera peut-être pas chez d'autres espèces éloignées pour lesquelles l'étude devra être refaite.

Mais bien d'autres facteurs, non étudiés ici, comme la nature de l'hôte, la nourriture, etc., ont une forte action sur la fécondité des trichogrammes. Ainsi, avant de comparer ce caractère chez 2 espèces et pour relever des différences qui ne soient pas seulement épigénétiques, il faut s'assurer le contrôle d'un maximum de composants du milieu.

Reçu le 17 novembre 1980. Accepté le 21 janvier 1981.

\section{RÉFERENCES BIBLIOGRAPHIQUES}

Ashley T. R., Gonzalez D., 1974. Effect of various food substances on longevity and fecundity of Trichogramma. Environm. Entomol., 3, 169-171.

Benoit M., 1977. Etude de l'incidence du vieillissement maternel sur la descendance fille de deux Lépidoptères : Epichorista ionophela Meyr. (Tortricidae) et Ephestia kuehniella Zell. (Pyralidae) et de leur parasite oophage : Trichogramma evanescens West. (Trichogrammatidae). Thèse du $3^{e}$ cycle, Université F. Rabelais de Tours, 110 pp.

Benoit M., Voegele J., 1979. Choix de l'hôte et comportement trophique des larves de Trichogramma evanescens (Hym. Trichogrammatidae) en fonction du développement embryonnaire de Ephestia kuehniella et Ostrinia nubilalis (Lep. : Pyralidae). Entomophaga, 24, 199-207.

Biemont C., 1978. Inbreeding effects : evidence for a genetic system which regulates viability in Drosophila melanogaster populations. Mech. Ageing Develop., 8, 21-42.

Biemont C., Bouletreau M., 1980. Hybridization and inbreeding effects on genome coadaptation in a haplo-diploïd hymenoptera : Cothonaspis boulardi (Eucoilidae). Experientia, 36, 45-46.

Cohet Y., Bouletreau-Merle J., 1974. Influences épigénétiques sur la reproduction d'un insecte : variation de la réactivité des femelles de Drosophila melanogaster à la copulation en fonction de leur température de développement. C.R. Acad. Sci., Paris, Série D, 278, 3235-3238.

David J., 1961. Influence de l'état physiologique des parents sur les caractères des descendants. Etude chez Drosophila melanogaster Meig. Ann. Genet. 3, 1-78.

David J., Bocquet Ch., Scheemaeker-Louis M. de, Pla E., 1978. Utilisation du coefficient de variation pour l'analyse de la variabilité de différents caractères quantitatifs chez Drosophila melanogaster : comparaison de souches appartenant à trois races géographiques. Arch. Zool. exp. gén., 118, 481-494.

David J., Cohet Y., Fouillet P., 1975. The variability between individuals as a measure of senescence. A study of the number of eggs laid and the percentage of hatched eggs in the case of Drosophila melanogaster. Exp. Gerontol., 10, 17-25.

Falconer D. S., 1974. Introduction à la génétique quantitative. Ed. Masson et Cie, Paris, 284 pp.
Flanders S. E., 1935. Effect of host density on parasitism. J. econ. Entomol., 28, 898-900.

Gerber H. S., Klostermeyer E. C., 1970. Sex control by bees : A voluntary act of egg fertilization during oviposition. Science, U.S.A., 167, 82-84.

Helle W., 1965. Inbreeding depression in an arrhenotokous mite (Tetranychus urticae Koch.). Entomol. exp. appl., 8, 299-304.

Hollingsworth M. J., Maynard-Smith J., 1955. The effects of inbreeding on rate of development and on fertility in Drosophila subobscura. J. Genet., 53, 295-314.

Kuno E., 1962. The effect of population density on the reproduction of Trichogramma japonicum Ashmead (Hymenoptera: Trichogrammatidae). Res. popul. Eco., 4, 47-59.

Lund H. O., 1938. Studies on longevity and productivity in Trichogramma evanescens. J. Agric. Res., 56, 421-439.

Nguyen Ban J., 1974. Elevage en laboratoire de trichogrammes en prévision d'une lutte intégrée contre Earias biplaga (Wlk). Note préliminaire. Café, Cacao, Thé, 18, 193-197.

Russo J., 1980. Caractérisation de quatre souches de trichogrammes (Hyménoptères, Trichogrammatidae) trouvées sur pontes d'Ostrinia nubilalis (Lépidoptères, Pyralidae). Thèse de $3^{\mathrm{c}}$ cycle, Université des Sciences et Techniques du Languedoc (Montpellier), $136 \mathrm{pp}$.

Voegele J., 1969. Les Hyménoptères parasites oophages des Aelia. Al Awamia, Rev. de la Rech. agron. maroc., 31, 137-323.

Voegele J., Daumal J., Brun Ph., Onillon J., 1975. Action du traitement au froid et aux ultra-violets de l'cuf d'Ephestia kuehniella (Pyralidae) sur le taux de multiplication de Trichogramma evanescens et $T$. brasiliensis (Hymenoptera : Trichogrammatidae). Entomophaga, 19, 341-348.

Watanabe T. K., Watanabe T., 1973. Fertility genes in natural populations of Drosophila melanogaster. III. Superiority of inversion heterozygotes. Evolution, 27, 468-475.

Wright S., 1931. Evolution in mendelian populations. Genetics, 16, 97-159.

Wright S., 1933. Inbreeding and homozygotie. Proc. Nat. Ac. Sc., 19, $411-420$. 